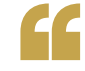

the SIV

reservoir is established within the first 3 days of infection

VIRAL INFECTION

\title{
Seeding the HIV-1 reservoir
}

HIV-1 can persist in latently infected memory CD4 ${ }^{+} \mathrm{T}$ cells, where it avoids clearance by antiretroviral therapy (ART). Despite the importance of this reservoir for viral rebound, little is known about how rapidly the reservoir is seeded and how this is affected by early ART. Now, Whitney et al. show that the viral reservoir is established within the first few days of infection, even before systemic viraemia is detected.

To model HIV-1 reservoir seeding, rhesus monkeys were infected with simian immunodeficiency virus (SIV) and ART was initiated at $3,7,10$ or 14 days post-infection. Notably, in animals that were treated on day 3, viral RNA was undetectable in plasma at all time points during the infection, whereas treatment that was initiated on day 7, day 10 or day 14 led to reduced viral replication and undetectable viraemia within 3-4 weeks of ART. The impact of ART on viraemia was consistent with adaptive immune responses: animals that were treated on day 3 lacked SIV-specific antibodies and SIV-specific T cell responses, whereas both humoral and cellular immune responses were detectable in animals that were treated on day 7 , day 10 or day 14. These findings suggest that early treatment impedes the establishment of viraemia, which correlates with the initiation of adaptive immunity.

The authors then examined whether the time of ART initiation had any effect on the viral reservoir by assessing the presence of proviral DNA in mononuclear cells from peripheral blood (PBMCs), inguinal lymph nodes (LNMCs) and the gastrointestinal mucosa (GMMCs). Animals that were treated on day 7 , day 10 or day 14 had lower levels of proviral DNA in PBMCs, LNMCs and GMMCs, compared with untreated animals. Importantly, although proviral DNA was not detected in the PBMCs of monkeys that were treated on day 3 , low levels of proviral DNA were found in the LNMCs and GMMCs, which indicates that the viral reservoir is seeded within the first 3 days of infection.

To investigate viral relapse, ART was discontinued on week 24 and all monkeys were monitored for the emergence of viraemia. Strikingly, although earlier initiation of ART correlated with a delayed onset of viral rebound, viral RNA was detected in the plasma of all animals,

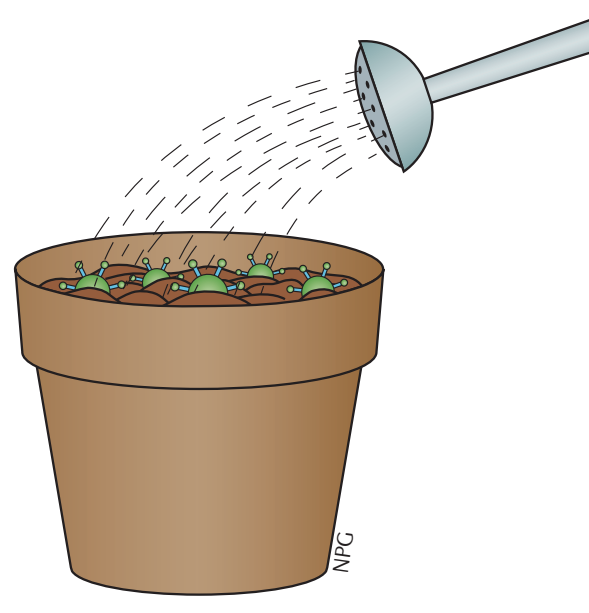

including those that initiated ART on day 3 and lacked systemic viraemia.

These data show that the SIV reservoir is established within the first 3 days of infection, which enables the virus to rebound after the discontinuation of ART. Furthermore, although there are important differences between the dynamics of SIV and HIV-1 infection, these data are consistent with clinical studies of humans infected with HIV-1. Therefore, this study indicates that early ART does not eliminate the viral reservoir and that it delays, but does not prevent, viral rebound following the discontinuation of ART, which has important implications for current treatment regimens.

Cláudio Nunes-Alves

ORIGINAL RESEARCH PAPER Whitney, J. B. et al. Rapid seeding of the viral reservoir prior to SIV viraemia in rhesus monkeys. Nature http://dx.doi. org/10.1038/nature13594 (2014) 